\title{
Creación de Empresa comercializadora y distribuidora de Paneles Solares en el Cantón Quevedo-Ecuador
}

\section{Creation of commercialization and distribution company of Solar Panels in Canton Quevedo-Ecuador}

\author{
*Rosa Karina Murillo Limonez ${ }^{1}$,**Gina del Pilar Rendón Guerra ${ }^{1}$,***Rudy Ivonne \\ Ortega Cabrera ${ }^{1}, * * * *$ Guido Marcelo Moreno Cedeño ${ }^{1}$ \\ ${ }^{1}$ Universidad Técnica Estatal de Quevedo, Facultad de Ciencias Empresariales \\ Campus Ing. Manuel Haz Alvares, km 1.5 vía Santo Domingo de Los Tsáchilas. Quevedo, \\ Los Ríos, Ecuador \\ *rmurillo@uteq.edu,***grendon@uteq.edu.ec,***rortega@uteq.edu.ec; \\ ****gmoreno@uteq.edu.ec
}

Fecha de recepción: 19/05/2018

Fecha de aceptación: 10/10/2018 Publicado: 26/12/2018

\section{Resumen}

El presente estudio se realizó en el Cantón Quevedo, se ubica en la zona central del país, posee una población de 173.575, habitantes. Después de determinar los elementos a considerar en la creación de una empresa de comercialización y distribución de paneles solares en el cantón Quevedo, provincia de Los Ríos, se procedió a seleccionar la información referente a: El estudio de mercado, a través de la determinación de la oferta, incluyó encuestas realizadas a los habitantes del cantón Quevedo. La demanda se obtuvo mediante encuestas dirigidas a personas que podrían ser los posibles compradores de dichos paneles. Los resultados muestran que el análisis de los datos recogidos en el estudio de mercado permiten determinar la factibilidad de poner en marcha la empresa en Quevedo, y pone de manifiesto la necesidad que tienen los demandantes de tener paneles solares para suplir la necesidad de energía suplementaria, además de conciencia ecológica. Referente al estudio técnico se evidencia el lugar destinado para la 
instalación de la empresa. La inversión requerida para el proyecto resultó ser de \$75,583.59, la cual un $70 \%$ será cubierto por financiamiento de una institución financiera y el 30\% restante por aporte propio. Finalizando los cálculos y análisis financieros se determinó la factibilidad del proyecto, ya que se obtiene un punto de equilibrio al primer año por $\$ 45,241.45$; VAN $\$$ 69,735.40; TIR 45\% y relación beneficio costo de 2.17.

Palabras Clave: Comercialización, Paneles Solares, distribución, Ecología, energía alternativa.

\begin{abstract}
This study was conducted in the Canton Quevedo of its geographic position, situated in the central part of the country, has a population of 173,575, inhabitants. After determining the elements to consider when starting a business marketing and distribution of solar panels in the canton Quevedo, Los Rios province, proceeded to select information regarding: The market, through the determination of the offer, included surveys of Quevedo Canton residents. The demand was obtained by surveys aimed at people who might be potential buyers of these panels. The results show that the analysis of the data collected in the market survey for determining the feasibility of launching the company in Quevedo, and highlights the need of the applicants have solar panels to supply the need of additional energy well as environmental awareness. Concerning the technical study evidenced the position intended for the installation of the company. The investment required for the project turned out to be $\$ 75,583.59$, which $70 \%$ will be covered by financing from a financial institution and the remaining 30\% own contribution. Completing the calculations and financial analysis project feasibility was determined as a balance to the first year, $\$ 45,241.45$ is obtained; VAN $\$ 69,735.40$; TIR $45 \%$ and cost benefit ratio of 2.17 .
\end{abstract}


Keywords: Commercialization, Solar paddles, distribution, Ecology, alternative energy.

\section{Introducción}

El Ecuador es un país privilegiado con respecto a condiciones meteorológicas y ubicación geográfica para la utilización de paneles solares. Primeramente al encontrarse en la latitud $0^{\circ}$ no existen variaciones representativas de horas efectivas de luz solar al día. En este país existe a lo largo de todo el año un promedio de 12 horas diarias de sol, variando entre alrededor de 11.45 a 12.20 horas dependiendo de la posición de la tierra en la órbita elíptica alrededor del sol. ANUARIO SOLAR, (2011). Un panel solar (o módulo solar) es un dispositivo que aprovecha la energía de la radiación solar. El término comprende a los colectores solares utilizados para producir agua caliente (usualmente doméstica) mediante energía solar térmica y a los paneles fotovoltaicos utilizados para generar electricidad mediante energía solar fotovoltaica. VILABOA, (2009). Un panel solar, de este modo, es un elemento que permite usar los rayos del sol como energía. Lo que hacen estos dispositivos es recoger la energía térmica o fotovoltaica del astro y convertirla en un recurso que puede emplearse para producir electricidad o calentar algo. El presente proyecto pretende determinar la factibilidad de comercializar paneles solares como medio de energía sustitutiva. Si bien la ciudadanía podría ser escéptica ante la introducción de este nuevo producto, tendría una nueva alternativa para solucionar una urgente necesidad (www.renova-energia.com/energia.../energia_solar_fotovoltaica.html, s.f.). Desde el punto de vista medioambiental, la energía fotovoltaica es favorable por ser limpio, exento de problemas de contaminación, producto de la combustión producido por los automotores, es decir beneficia la atmósfera, el suelo, el agua, la fauna, la vegetación, entre otros. Cómo influye la creación de una empresa de comercialización y distribución de paneles solares en el Cantón Quevedo. El proyecto 
es ambicioso, y sin duda beneficiará a la colectividad y al país en general en síntesis, el proyecto trata de la distribución e implementación de paneles solares como método de energía alternativa, sustentado en el fomento de una cultura para el uso racional de energía, enmarcado en el cambio de la matriz energética del Plan Nacional del Buen Vivir (www.buenvivir.gob.ec/, s.f.). Las preguntas de investigación planteadas tenemos: ¿Cómo influye una investigación de mercado para identificar la demanda de paneles solares?, ¿De qué manera el estudio técnico, organizacional y económico me permitirá conocer la estructura y monto de inversión para la creación de una empresa comercializadora de paneles solares?, ¿Cómo el estudio económico financiero permitirá determinar la rentabilidad y el tiempo de recuperación de la inversión que demanda la creación de una empresa comercializadora de paneles solares?. El objetivo principal es crear una empresa para la comercialización y distribución de paneles solares en el Cantón Quevedo.

\section{Materiales y Métodos}

\section{Localización y Duración de la Investigación}

El presente estudio se lo realizó en el Cantón Quevedo que por su posición geográfica, se ubica en la zona central del país, posee una población de 173.575, habitantes, según datos estadístico INEC censo 2010, su ubicación astronómica se encuentra entre las coordenadas de $71^{\circ} 20^{\prime} 30^{\prime \prime}$ de latitud sur y de $79^{\circ} 28^{\prime} 30^{\prime \prime}$ de latitud oeste, a una altitud promedio de 74 m.s.n.m. La duración de esta investigación fue aproximadamente 90 días.

\section{Métodos}




\section{Histórico- Lógico Descriptivo- Sistémico.}

Este método se lo aplicó para analizar los hechos sobre la oferta y demanda de paneles solares. Deductivo.- Se lo utilizó en el análisis lógico de la información general para implantar el plan de negocio en la creación de la empresa. Analítico - Sintético.- Porque éste método hace posible la comprensión de todo hecho, fenómeno, idea, caso, etc, es decir, permitió examinar la información en relación a la investigación. Mientras que la síntesis ayudó al análisis obtenido por medio de la investigación de campo operativa y por fuentes de investigación para tener como resultado una información clara y detallada

\section{Fuentes}

Para cumplir con los objetivos de la investigación se utilizó las siguientes fuentes. Primaria.- Para desarrollar la fuente primaria en la presente investigación se realizó encuestas a los habitantes del cantón Quevedo. Secundaria.- El uso de la fuente secundaria permitió obtener más información especializada de esta investigación la cual se recopiló mediante consultas de libros, revista, internet, folletos, relacionados con el tema de la presente investigación.

\section{Técnicas e instrumentos de evaluación}

En la presente investigación se utilizó las siguientes técnicas e instrumentos. Encuesta se encuestó a hombres y mujeres del área urbana del cantón Quevedo para determinar sus requerimientos en materia de paneles solares.

\section{Tipos de Investigación}

\section{De campo}

La investigación de campo permitió reunir la información necesaria mediante el proceso de la aplicación del método científico que procura obtener información relevante, para entender, 
verificar, corregir o aplicar el conocimiento, y permitir tener contacto con la realidad y mejorar los conocimientos de la investigación y así poder solucionar los problemas. Descriptiva.- Se aplicará un procedimiento positivo con el objeto de ir plasmando toda la información en datos resumidos y reales para utilizarla en la creación de una comercializadora de paneles solares. Bibliográfica.- Suministró información, tomada de libros, revistas, folletos, e internet, donde se exploró lo que se ha escrito en la comunidad científico sobre determinado tema o problema que hay que consultar, y como resolverlo, define cuestiones generales como el tema, el problema, etc. De este modo ayudó a reforzar la propuesta a investigar.

\section{Población y muestra}

\section{Población}

Quevedo tiene 173.575 habitantes de acuerdo al último censo de población y vivienda realizado en el 2010. Pero la población que se ha tomado en cuenta para obtener la muestra es la de 31.953 que es el número de casas que hay en Quevedo y son las personas de estas casas las que se beneficiarían con este producto.

\section{Muestra}

Para determinar el tamaño de la muestra a encuestar, se utilizó la siguiente fórmula:

$$
\begin{gathered}
n=\frac{Z^{2} * p^{*} q^{*} N}{E^{2}(N-1)+p^{*} q^{*} Z^{2}} \\
\text { n= Tamaño de la muestra } \\
\mathrm{N}=\text { Población a investigarse }=31.953 \\
\mathrm{Z}=\text { Nivel de confianza }=1.96(95 \%) \\
\mathrm{p}=\text { Variabilidad positiva }=50 \%
\end{gathered}
$$




$$
\begin{aligned}
& \mathrm{q}=\text { Variabilidad negativa }=50 \% \\
& \mathrm{E}=\text { precisión o error }=5 \% \\
& n=\frac{(1.96)^{2} *(0.5) *(0.5) *(31.953)}{(0.05)^{2}(31.953-1)+0.5 * 0.5 *(1.96)^{2}}
\end{aligned}
$$

Tamaño de la muestra $=379$ familias

\section{Resultados y Discusión}

En esta parte mostraremos los resultados obtenidos en la investigación de mercado, la parte técnica y financiera de la investigación.

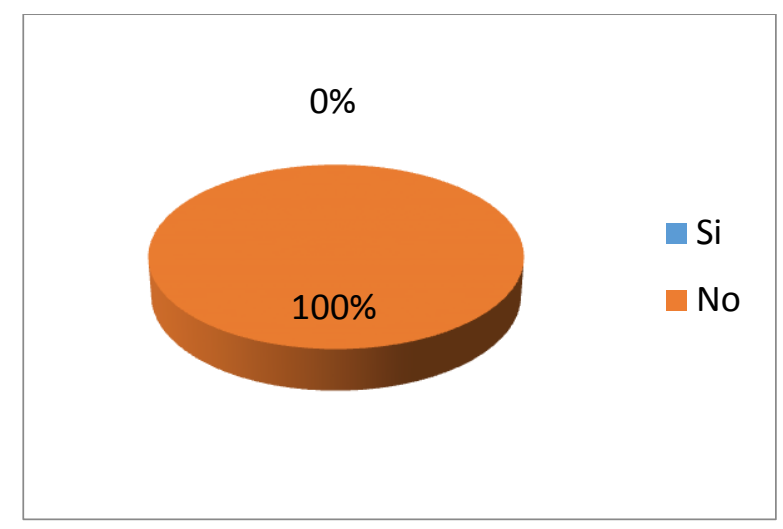

Figura 1. Utilización de Paneles Solares

En la Figura 1 podemos observar que el 100\% de los encuestados no usan paneles solares en el Cantón Quevedo.

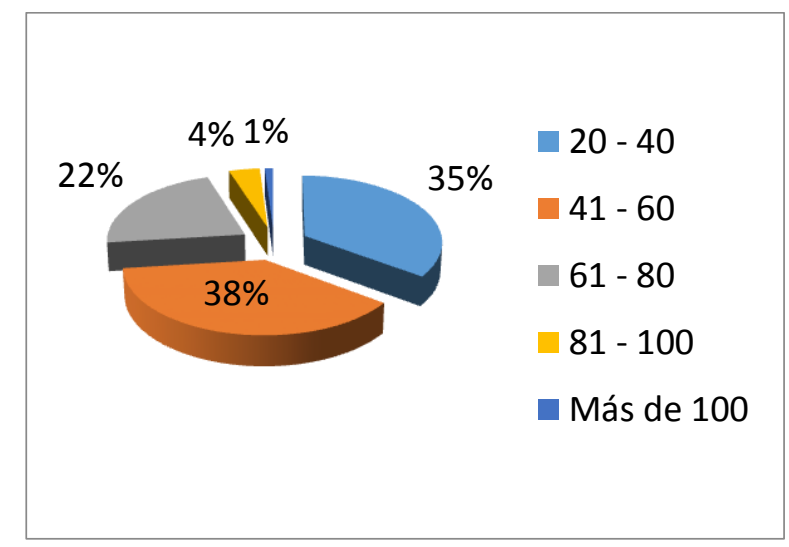


Figura 2. Consumo de energía mensual

En la Figura 2 se puede ver que el $38 \%$ de los encuestados expresan que su consumo de energía mensual es de 41 a 60 dólares; mientras que el 35\% de 20 a 40 mensuales; el $22 \%$ de 61 a 80 mensuales; el 4\% de 81 a 100 mensuales y el 1\% más de 100 dólares mensuales.

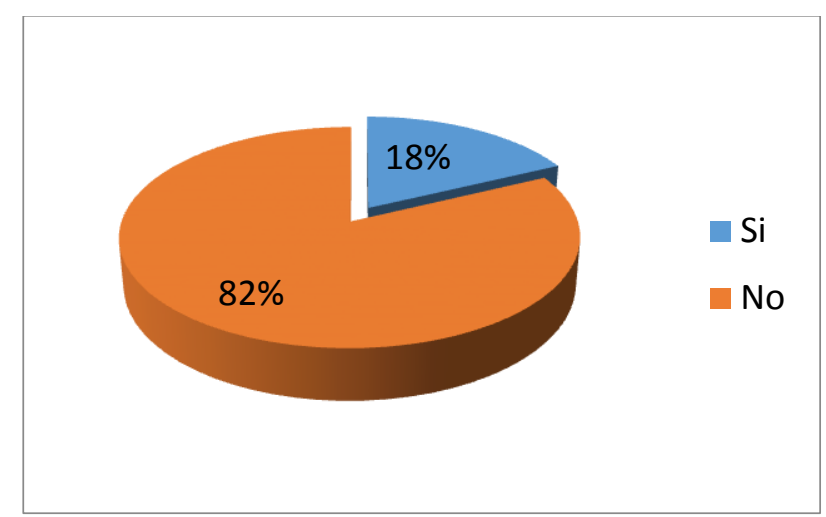

Figura 3. Conocimiento sobre paneles solares

En la Figura 3 se detalla que el $82 \%$ de los encuestados no han escuchado hablar sobre los paneles solares y el $18 \%$ restante sí.

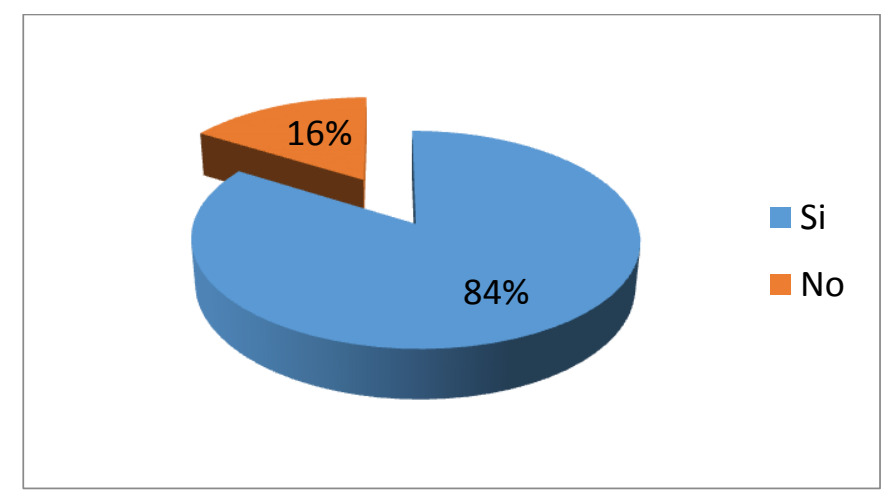

Figura 4. Adquisición de paneles solares

En la Figura 4 se detalla que el $84 \%$ de los encuestados si estarían dispuesto a adquirir paneles solares y el $16 \%$ restante no desean adquirir este producto. 
Revista Ciencias Sociales y Económicas - UTEQ (2018)

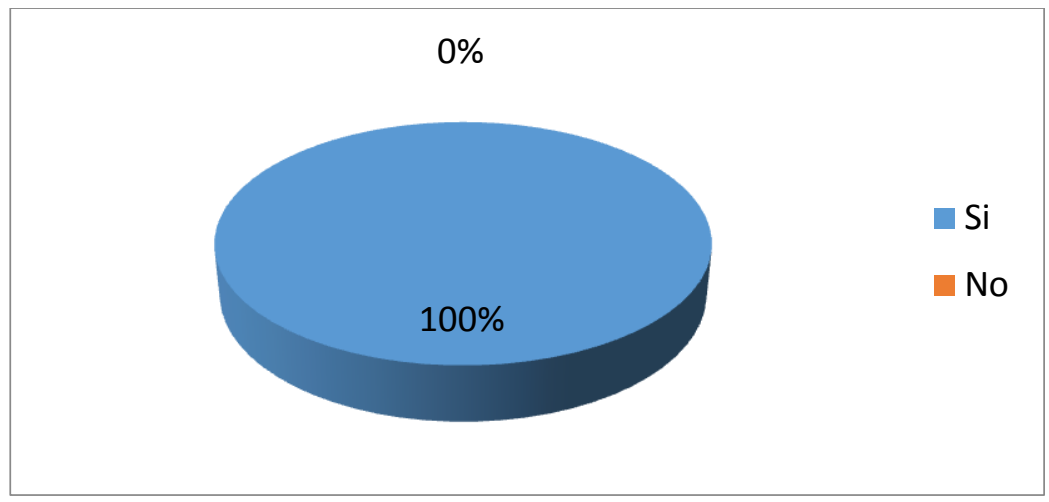

Figura 5. Evitaría la contaminación del ambiente utilizando energía limpia y renovable El $100 \%$ de los encuestados sí estarían dispuestos a evitar la contaminación del ambiente utilizando energía limpia y renovable.

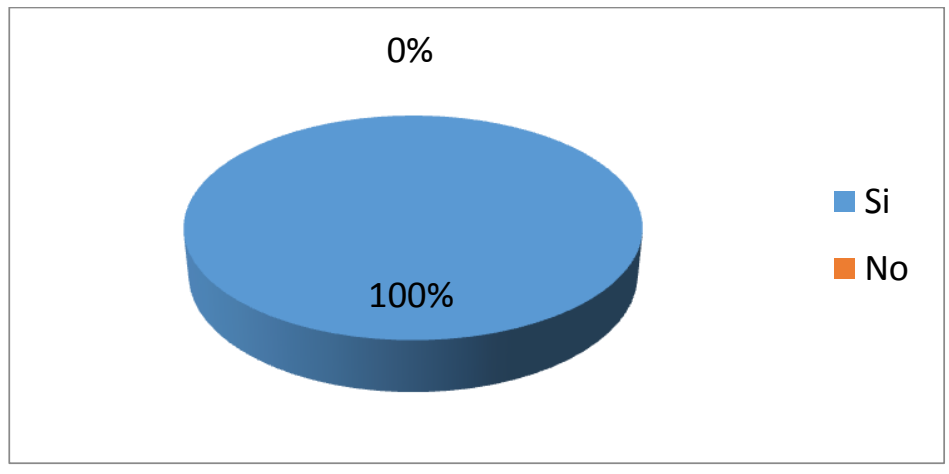

Figura 6. Empresa comercializadora de paneles solares

El 100\%, según la Figura 6, los encuestados si les gustaría una empresa que comercialice paneles solares. 
Revista Ciencias Sociales y Económicas - UTEQ (2018)

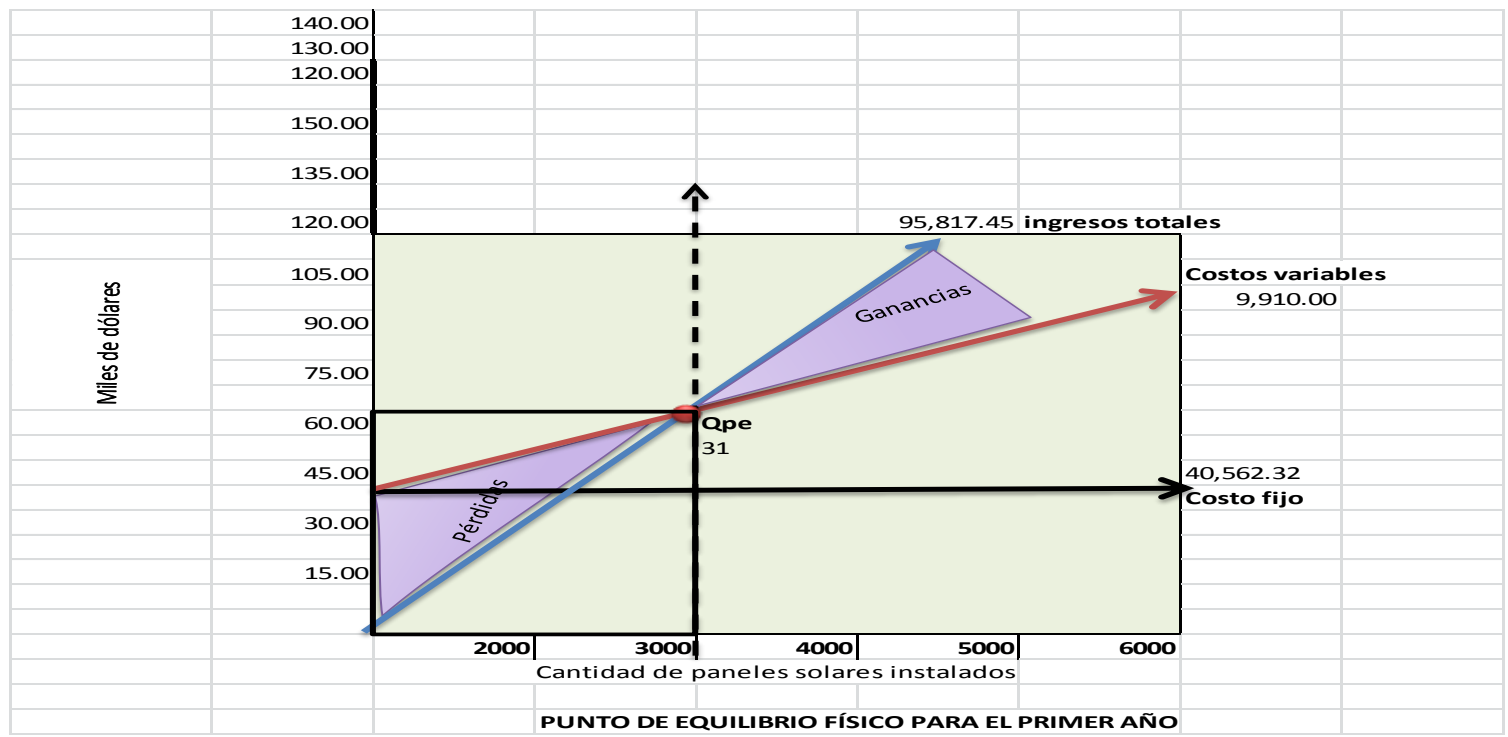

Figura 7.- punto equilibrio

El nivel de equilibrio monetario del primer año es de $\$ 45.241,45$ con lo cual la empresa "SUNTOB", ni gana ni pierde y el punto de equilibrio en unidades producidas es 31 paneles comercializados.

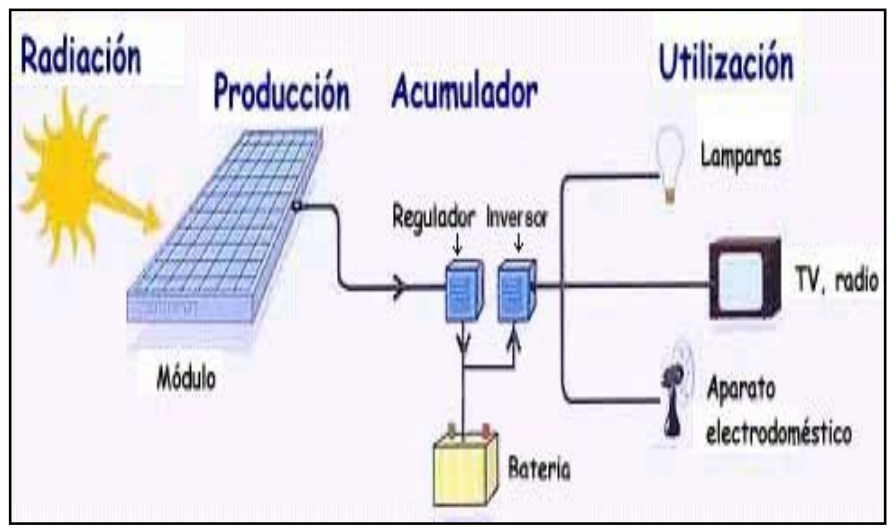

Figura 8. Estructura de un panel solar

\section{Discusión}


De las encuestas realizadas se pudo determinar que existe demanda dentro de este cantón tal como lo describe Baca (2008). La demanda se define como la cantidad y calidad de bienes y servicios que pueden ser adquiridos a los diferentes precios del mercado por un consumidor (demanda individual) o por el conjunto de consumidores. Este estudio de mercado se acopla a lo emitido por Mariotti (2008). En un estudio de factibilidad el estudio de mercado sirve como antecedente para la realización de los estudios técnicos, de ingeniería, financiera y económica para determinar la viabilidad de un negocio. En el estudio técnico se planteó el flujograma de procesos en la comercialización de paneles solares, de igual manera permitió visualizar el micro y macro localización de la futura empresa, por su parte Contreras (2008). La escogencia de la tecnología define en gran medida el resultado económico del proyecto, de acuerdo con la magnitud del proyecto es necesario adelantar los estudios de detalle en la localización general, construcciones civiles, mecánicas y eléctricas, para con base en ellos preparar los presupuestos de construcción. Dentro de lo posible el método general del diseño que debe primar es de funcionalidad técnica. En el estudio económico, la inversión de los activos fijos tangibles es 44,135.00. Baca (2008). Es el gasto dedicado a la adquisición de bienes que no son de consumo final, bienes de capital que sirven para producir otros bienes. En un sentido más amplio la inversión es el flujo de dinero que se encamina a la creación o mantenimiento de bienes de capital y a la realización de proyectos que presumen lucrativos.

En esta inversión se detalla los gastos de organización, pre-operativos dando un total de activos fijos intangibles de $\$ 2,000.00$ dólares y un capital de trabajo de $\$ 29,448.59$. El ingreso generado para el primer año de operatividad del proyecto es $\$ 95,817$; Wander (2011). Es la cantidad que se dispone para gastos de consumo personal y ahorros. También es proveniente de sueldos, salarios, dividendos, rentas, intereses, negocios, etc. En los estados financieros se 
observa la utilidad neta, luego de descontar todos los costos y gastos que se incurren cada año de vida útil del proyecto. Para el primer año la utilidad es de $\$ 30,063.82$, Tarrágo (2008). Son el producto final del proceso contable de la información financiera, elemento imprescindible para que los diversos usuarios puedan tomar decisiones: La información financiera que dichos usuarios requieren que se centre primordialmente en la evaluación de la situación financiera de la rentabilidad y la liquidez.

\section{Conclusiones}

El análisis de los datos recogidos en el estudio de mercado permite determinar la factibilidad de poner en marcha la empresa en Quevedo. La inversión fija es de \$44,135.00; la inversión intangible de $\$ 2,000.00$ y un capital de trabajo de $\$ 29,448.59$. El proyecto resultó de $\$ 75,583.59$, la cual un $70 \%$ será cubierto por financiamiento de una institución financiera y el 30\% restante por aporte propio. Finalizando los cálculos y análisis financieros se determinó la factibilidad del proyecto, ya que se obtiene un punto de equilibrio al primer año por $\$ 45,241.45$; VAN $\$$ 69,735.40; TIR 45\% y relación beneficio costo de 2.17.

\section{Referencias Bibliográficas}

Anuario, S. 2011. Corporación de Desarrollo Tecnológico, Cámara Chilena de la Construcción. Primera edición. Pp. 152. Chile

Baca, G. 2008. Evaluación de proyectos. Quinta edición. McGraw-Hill, Madrid México. Pp. 274

Bermejo, M. 2008. El plan de negocio debe constituir la materialización de toda la planificación, 3era edición. Pp. 56. Colombia.

Cañas, B. 2008. Manual para formulación evaluación y ejecución de proyectos, $4^{\mathrm{a}}$. Edición, Pp.236. El Salvador. 
Careaga, P. 2008. Fundamentos de preparación y evaluación de proyectos de inversión.

Segunda Edición. Pp. 345. Bogotá - Colombia.

Chiavenato Idalberto, Introducción a la teoría general de la administración, 7a. ed., McGraw-

Hill Interamericana, 2008, p. 10.

Díez de Castro, E. 2008. Distribución comercial. Segunda edición. Segunda impresión. McGrawHill / Interamericana S.A. Madrid. España. Pp. 312

Guía práctica para crear o mejorar tu pequeña y mediana empresa, tu propio negocio 6, año 2012, El Diario Hoy.

Institute of Industrial Engineers, "Más allá de la Reingeniería", CECSA, México, 2007, p.4.

Kotler, P. 2007. Marketing estratégico. Editorial Pearson. Educación. Pág. 44. Colombia

Martín, W. 2008. Formulación y evaluación de proyectos tecnológicos empresariales aplicados. Serie gestores tecnológicos. Edición del Convenio Andrés Bello. Primera edición. Pp. 216. Colombia.

Starosta, Guido y KicilloF, Axel (2007): “On Materiality and Social Form: A Political Critique of Rubin's Value-Form Theory", Historical Materialism, Volume 15, Issue 3, 2007, ISSN: 1465-4466, pp. 9-43;

Varela, R. 2010. Innovación empresarial. Editorial Prentice Hall. Pág. 23-28. Chile

Vilaboa, M. 2009 “Análisis y Caracterización del Mercado de los Paneles Solares en Chile”. DII Universidad de Chile. Primera edición. Pp. 28. Chile www.buenvivir.gob.ec/. (s.f.). Recuperado el 23 de 04 de 2015, de www.buenvivir.gob.ec/ www.renova-energia.com/energia.../energia_solar_fotovoltaica.html. (s.f.). Recuperado el 23 de 04 de 2015, de www.renova-energia.com/energia.../energia_solar_fotovoltaica.html 